\title{
EFECTO DEL TOLTRAZURIL Y LA COMBINACIÓN DE SULFADOXINA Y PIRIMETAMINA EN EL TRATAMIENTO DE LA SARCOCISTIOSIS CANINA DURANTE EL PERIODO PATENTE
}

\author{
EFFECT OF TOLTRAZURIL AND THECOMBINATION OF SULFADOXINE AND PIRIMETAMINE IN THE \\ TREATMENT OF CANINE S A R COCYSTIOSIS DURING THE PREPATENT PERIOD
}

\author{
María Barrientos V. ${ }^{1}$, Amanda Chávez V. ${ }^{2}$, Atilio Pacheco P. ${ }^{3}$, Daniel Ticona S. ${ }^{4}$ y \\ Víctor Leyva V. ${ }^{5}$
}

\section{Resumen}

El objetivo del estudio fue evaluar la eficacia del toltrazuril (2.5\%) y de la combinación de sulfadoxina y pirimetamina $(500 / 25 \mathrm{mg}$ ) en el tratamiento de la sarcocistiosis canina. El estudio se realizó durante los meses de octubre del 2005 a marzo del 2006, en la Estación Experimental del Centro de Investigaciones IVITA, Maranganí, Cusco, perteneciente a la Facultad de Medicina Veterinaria, UNMSM. Se utilizó 15 cachorros cruzados de ambos sexos, de 2-3 meses de edad, previamente desparasitados y alimentados solo con concentrado. Todos ellos fueron infectados con aproximadamente 9,000-10,000 quistes de Sarcocystis lamacanis, presentes en 4-5 g de tejido cardiaco de alpaca. Las heces se analizaron diariamente hasta observar la presencia de esporoquistes u ooquistes de Sarcocystis sp. Posteriormente fueron distribuidos en tres grupos de 5 animales c/u. Un grupo control no tratado y dos grupos tratados diariamente por 10 días, uno con toltrazuril (2.5\%) en dosis de $15 \mathrm{mg} / \mathrm{kpv}$ y otro grupo con la combinación de sulfadoxina y pirimetamina (500/25 mg) en dosis de 20 y $1 \mathrm{mg} / \mathrm{kgpv}$, respectivamente. Muestras fecales fueron recolectadas diariamente y se analizaron por el método de coproparasitologico de flotación con solución de Sheather para determinar la presencia de ooquistes o esporoquistes de Sarcocystis sp., y las muestras positivas se cuantificaron por el método de Stoll modificado, para determinar su número por gramo de heces. Los resultados muestran que el toltrazuril alcanzó una eficacia en el control de la sarcocistiosis del 94.7 $\%$ al tercer día de tratamiento y un $100 \%$ al sexto día, mientras que la combinación de sulfadoxina y pirimetamina no logró controlarla, llegando a mostrar una moderada eficacia al segundo día post tratamiento $(88.1 \%)$.

Palabras clave: Toltrazuril, sulfadoxina, pirimetamina, sarcocistiosis, efectividad

\footnotetext{
${ }^{1}$ Práctica privada

${ }^{2}$ Laboratorio de Microbiología y Parasitología Veterinaria, FMV-UNMSM

E-mail: a_chavez_g@hotmail.com

${ }^{3}$ Laboratorio de Sanidad Animal, FAZ-UNSAAC

${ }^{4}$ Estación Experimental del Centro de Investigaciones IVITA-Maranganí, FMV-UNMSM

${ }^{5}$ Laboratorio de Reproducción Animal, FMV-UNMSM
} 


\section{Abstract}

The objective of this study was to evaluate the efficacy of toltrazuril (2.5)\% and the combination of sulfadoxine and pirimetamine $(500-25 \mathrm{mg}$ ) on the treatment of canine sarcocystiosis. The study was conducted from October 2005 until March 2006 in the Experimental Station of the IVITA Research Center, Maranganí, Cusco. A total of 15 crossbred puppies, both sexes, 2-4 months of age, that were dewormed and fed with a diet extent of meat were used. Puppies were infected with approximately 9,00010,000 cysts of Sarcosystis lamacanis present in 4-5 g of alpaca heart. Feces were daily collected and analyzed to observe the presence of sporocysts or oocysts. Then, were distributed in three groups of 5 animals each. One group remained untreated (as control) and the other two groups were daily treated during 10 days with $15 \mathrm{mg} / \mathrm{kg}$ of toltrazuril (2.5\%) or the combination of sulfadoxine and pirimetamine $(500-25 \mathrm{mg})$ in a dose of 20 and $1 \mathrm{mg} / \mathrm{kg}$ respectively. Fecal samples were daily collected and analyzed by the flotation method using the Sheather solution to determine the presence of sporocysts or oocysts and the modified Stoll method to quantify the number of eggs per gram of feces. The results showed that the efficacy of toltrazuril in the control of sarcosystiosis was $94.7 \%$ at the $3^{\text {rd }}$ day of treatment and $100 \%$ at day 6 , whereas the combination of sulfadoxine and pirimetamine did not control it, and only a moderate efficacy $(88.1 \%)$ was observed in the $2^{\text {nd }}$ day.

Key words: toltrazuril, sulfadoxine, pirimetamine, sarcosystiosis, effectiveness

\section{INTRODUCCIÓN}

El Perú cuenta con alrededor de 3’192,870 alpacas (más del $90 \%$ de la población mundial) y 1'130,543 de llamas a nivel nacional (INIA, 2007). La producción de fibra de alpaca tiene un importante atractivo comercial (Corrales, 2000); además, la carne de alpaca y llama tiene un alto valor proteico y es un importante recurso nutricional que debe ser aprovechado por el país.

No obstante las ventajas que presentan estas especies, su desarrollo se encuentra limitada por diversos factores que inciden negativamente en los niveles productivos. Dentro de estos, las enfermedades parasitarias tienen un rol preponderante (Bustinza, 2000), especialmente la sarcocistiosis ocasionada por el Sarcocystis sp., protozoario perteneciente al grupo de los apicomplexos, que poseen un ciclo de vida indirecto, y donde los carnívoros son los hospederos definitivos (perros, zorros, etc.) y los herbívoros (camélidos suda- mericanos, bovinos, ovinos, etc.) actúan como hospederos intermediarios (Leguía y Casas, 1999).

La sarcocistiosis disminuye la productividad de los camélidos sudamericanos (CSA), ya que afecta en forma masiva las fibras musculares esqueléticas, cardiacas y viscerales al formar quistes macroscópicos (S. aucheniae) y microscópicos ( S. lamacanis) (Leguía y Clavo, 1989). Esto ocasiona fuertes pérdidas debido al decomiso en los camales de la carne afectada, que se estima en $\$ 300,000$.00 dólares anuales (Leguía, 1991), aparte de la baja aceptación de esta carne en los mercados (Corrales, 2000).

Los pequeños productores y las comunidades campesinas, pobladores alto andinos de bajos niveles económicos y culturales, poseen más del $80 \%$ de las alpacas y la casi totalidad de llamas (Franco et al., 1998), lo que contribuye a perpetuar esta parasitosis, permitiendo altas prevalencias, que en muchos casos alcanza el 100\% de los animales mayores de 2 años de edad (Mostajo, 1983). 
Actualmente, se vienen realizando estudios dirigidos principalmente a detener el ciclo biológico en el hospedero definitivo, ya que los cánidos, luego de consumir las vísceras crudas infectadas, eliminan millones de esporoquistes en el medio ambiente y de esa forma, infectan a los CSA, completando el ciclo biológico de este parásito.

Como parte de las actividades de investigación encaminadas al control de la sarcocistiosis, se han evaluado drogas como el Ponazuril (Lindsay et al., 2000). En el Perú, la Universidad Nacional del Altiplano de Puno ha evaluado varios tratamientos contra la sarcocistiosis intestinal en caninos utilizando combinaciones de sulfadoxina y pirimetamina, así como la primaquina, obteniendo el $100 \%$ de eficacia luego de un tratamiento continuo por un periodo de 7 ó más días (Saravia, 2003; Yujra, 2004). Sin embargo, el esquema de desparasitación, aunado a su elevado costo, ha impedido que pueda ser aplicado en forma rutinaria.

Otras drogas anticoccidiales existentes en el mercado como el toltrazuril han tenido efecto en todos las fases endógenas y han demostrado ser eficaces en aplicaciones únicas. Daugschies et al. (2000) usaron el toltrazuril en dosis de 10, 20 ó $30 \mathrm{mg} / \mathrm{kg}$ de peso vivo en perros infectados experimentalmente y de forma natural con Cystoisospora, logrando inhibir la eliminación de esporoquistes. El presente estudio evalúa la eficacia de un tratamiento con toltrazuril al $2.5 \%$ y otro con la combinación de sulfadoxina y pirimetamina $(500 / 25 \mathrm{mg}$ ) para el tratamiento de la sarcocistiosis canina.

\section{Materiales y Métodos}

\section{Ubicación del estudio}

El estudio se realizó en la Estación Experimental del Centro de Investigación IVITAMaranganí, a una altitud de 3,709 msnm, en la provincia de Canchis, región Cusco, de la Facultad de Medicina Veterinaria, Universidad Nacional Mayor de San Marcos.

\section{Manejo de los canes}

Se utilizaron 15 cachorros cruzados de ambos sexos, de aproximadamente 2-3 meses de edad. Los animales fueron desparasitados y permanecieron en observación por dos semanas antes del inicio del experimento. Recibieron alimento balanceado comercial, libre de carne cruda, y se les suministró agua ad libitum.

Los animales fueron alojados en casetas individuales (caniles) de madera, con dimensiones de 1.0 x 0.80 x $0.90 \mathrm{~m}$. Las casetas se lavaron y desinfectaron con lejía, kreso $\mathrm{y}$ formol antes de introducir a los cachorros. El piso de las jaulas fue cubierto con papel periódico, el cual fue cambiado dos veces al día.

Se utilizaron corazones de alpacas con microquistes de $S$. lamacanis, los cuales fueron obtenidos del camal de Sicuani. Los cachorros fueron infectados por vía oral, con 9,000-10,000 microquistes presentes en 4-5 $\mathrm{g}$ de tejido cardiaco.

\section{Procedimiento experimental}

A través de un diseño completamente al azar, los cachorros que se encontraban en el día 6 a 8 del inicio del periodo patente (fase de eliminación de ooquistes y esporoquistes de $S$. lamacanis) fueron distribuidos en tres grupos de 5 animales cada uno:

- Grupo A: Controles no tratados.

- Grupo B: Tratado con toltrazuril al $2.5 \%$ en dosis de $15 \mathrm{mg} / \mathrm{kg}$ de peso vivo.

- Grupo C: Tratado con un comprimido comercial de sulfadoxina de $500 \mathrm{mg}$ y pirimetamina de $25 \mathrm{mg}$, usando una dosis de 20 y $1 \mathrm{mg} / \mathrm{kg}$ de peso vivo, respectivamente.

La dosis de sulfadoxina y pirimetamina se basó en la posología recomendada por Madison et al. (2004). Durante el periodo prepatente se administraron los fármacos por vía oral, en ayunas, y con la ayuda de una jeringa hipodérmica sin aguja, por 10 días consecutivos. 
Se colectaron muestras fecales a partir del día 10 post-infección y hasta el término del experimento. El análisis coprológico se hizo mediante el método de concentración por flotación con solución azucarada o de Sheather con el objetivo de determinar la presencia de esporoquistes en heces. Las muestras positivas fueron cuantificadas mediante el método de Stoll modificado para determinar el número de esporoquistes por gramo de heces.

Todos los cachorros infectados con corazón de CSA domésticos conteniendo microquistes de S. lamacanis completaron su periodo prepatente a los 14 días post-infección; es decir, se evidenció la presencia de esporoquistes en sus heces al día 18 ó 20 post-infección. Posterior a ello se procedió al tratamiento de los animales, considerándose como día cero el día previo al tratamiento.

La eficacia de los tratamientos se determinó mediante la siguiente fórmula:

\section{Eficacia $\%=\underline{\text { PEGC }- \text { PEGT }} \times 100$ PEGC}

donde:

PEGC $=$ Promedio de esporoquistes del grupo control

PEGT $=$ Promedio de esporoquistes del grupo tratado

La eficacia se clasificó de la siguiente forma (Kassai, 1998):

- Muy eficaz (>98\%)

- Eficaz (90-98\%)

- Moderadamente eficaz (80-89\%)

- Insuficientemente activo $(<80 \%)$

\section{Análisis estadístico}

La variable número de esporoquistes por gramo de heces (epg) de cada tratamiento se analizó bajo un arreglo factorial de 2 × 2 mediante el diseño de bloque completo al azar.
Las diferencias entre grupos de tratamiento (número de esporoquistes) se determinaron mediante análisis de varianza. Se utilizó la prueba de Tukey (Daniel, 1996) para determinar diferencias entre promedios.

\section{Resultados}

Los cachorros tratados con toltrazuril presentaron una reducción significativa de epg a partir del $3^{\text {er }}$ día de tratamiento en comparación con el grupo control, y a partir del $6^{\circ}$ día se logró controlar la infección parasitaria. Por otro lado, los cachorros tratados con sulfadoxina y pirimetamina mantuvieron, con ligeras variaciones, la carga parasitaria durante el tiempo que duró la evaluación (Cuadro 1).

El Cuadro 2 muestra la eficacia de los fármacos bajo evaluación en el control de la sarcocistiosis del canino. La eficacia del toltrazuril superó el $90 \%$ al $3^{\text {er }}$ día y alcanzó el $100 \%$ al $6^{\circ}$ día del tratamiento, mientras que la combinación de sulfadoxina y pirimetamina tuvo una eficacia moderada.

\section{Discusión}

Se vienen realizando esfuerzos para controlar la sarcocistiosis en CSA. Entre éstos, se encuentra el empleo de una vacuna en el hospedero intermediario (A. Hung, comunicación personal), así como el uso de fármacos que actúan sobre las fases sexuales de esta coccidia (gametogonia) presentes en el hospedero definitivo (Saravia, 2003; Yujra, 2004).

Se conoce que el toltrazuril actúa sobre las fases asexuales (esquizogonia) y sexuales (gametogonia) de todas las eimerias (Mehlhorn et al., 1988, citado por Marthis et $a l ., 2004)$. Este fármaco ha sido utilizado con éxito en el control de eimeria de aves cuando 
Cuadro 1. Efecto del toltrazuril y de la combinación de sulfadoxina y pirimetamina, expresado como número de esporoquistes por gramo de heces (epg), en el control de la sarcocistiosis canina (Sarcocystis lamacanis)

\begin{tabular}{cccc}
\hline \multirow{2}{*}{$\begin{array}{c}\text { Días post- } \\
\text { tratamiento }\end{array}$} & \multicolumn{3}{c}{ Tratamientos } \\
\cline { 2 - 4 } Control & $\begin{array}{c}\text { Toltrazuril al } 2.5 \% \\
(15 \mathrm{mg} / \mathrm{kg})\end{array}$ & $\begin{array}{c}\text { Sulfadoxina }(20 \mathrm{mg} / \mathrm{kg}) \mathrm{y} \\
\text { Pirimetamina }(1 \mathrm{mg} / \mathrm{kg})\end{array}$ \\
\hline 1 & $880^{\mathrm{a}}$ & $320^{\mathrm{a}}$ & $680^{\mathrm{a}}$ \\
2 & $920^{\mathrm{a}}$ & $320^{\mathrm{a}}$ & $560^{\mathrm{a}}$ \\
3 & $1720^{\mathrm{a}}$ & $40^{\mathrm{b}}$ & $320^{\mathrm{b}}$ \\
4 & $1560^{\mathrm{a}}$ & $80^{\mathrm{b}}$ & $1400^{\mathrm{a}}$ \\
5 & $1880^{\mathrm{a}}$ & $40^{\mathrm{b}}$ & $1240^{\mathrm{ab}}$ \\
6 & $2380^{\mathrm{a}}$ & $0^{\mathrm{b}}$ & $1040^{\mathrm{ab}}$ \\
7 & $2200^{\mathrm{a}}$ & $0^{\mathrm{b}}$ & $960^{\mathrm{ab}}$ \\
8 & $2400^{\mathrm{a}}$ & $0^{\mathrm{b}}$ & $1000^{\mathrm{ab}}$ \\
9 & $1760^{\mathrm{a}}$ & $0^{\mathrm{b}}$ & $760^{\mathrm{ab}}$ \\
10 & $2040^{\mathrm{a}}$ & $0^{\mathrm{b}}$ & $840^{\mathrm{ab}}$ \\
\hline Total & 17740 & 800 & 8800 \\
\hline a,b Letras diferentes dentro de filas indican diferencias significativas $(\mathrm{p}<0.05)$
\end{tabular}

Cuadro 2. Eficacia ${ }^{1}$ de toltrazuril $(2.5 \%)$ y de la combinación de sulfadoxina $(20 \mathrm{mg} / \mathrm{kg})$ y pirimetamina $(1 \mathrm{mg} / \mathrm{kg})$ en el control de sarcocistiosis intestinal en caninos infectados experimentalmente con S. lamacanis

\begin{tabular}{lcccccccccc}
\hline & \multicolumn{10}{c}{ Días de tratamiento } \\
\cline { 2 - 11 } & 1 & 2 & 3 & 4 & 5 & 6 & 7 & 8 & 9 & 10 \\
\hline Toltrazuril & 63.6 & 65.2 & 97.7 & 94.8 & 97.8 & 100 & 100 & 100 & 100 & 100 \\
$\begin{array}{l}\text { Sulfadoxina y } \\
\text { Pirimetamina }\end{array}$ & 70.5 & 85.1 & 88.1 & 55.6 & 41.0 & 59.0 & 59.6 & 66.1 & 65.8 & 65.4 \\
\hline 1
\end{tabular}

${ }^{1}$ Expresado en porcentaje

se administró por dos días (Marthis et al., 2004), así como en el tratamiento de eimerias e isosporas de algunos mamíferos (Daugschies et al., 2000; Lloyd y Smith, 2001; Mundt et al., 2003). En el presente estudio la administración del toltrazuril al $2.5 \%$ produjo una marcada reducción de la carga de esporoquistes de $S$. lamacanis en las heces a partir del $3^{\text {er }}$ día de tratamiento. Sin embargo, este fármaco no pudo controlar una infección experimental de un estudio previo donde se le empleó con una dosis única de 10 ó 20 $\mathrm{mg} / \mathrm{kg}$ en los días 5 ó 7 post-infección (fase prepatente de la infección) (Vilca et al., 2007). 
Los resultados logrados con el toltrazuril son alentadores; sin embargo, su aplicación por 3 ó 6 días consecutivos no son prácticos para la realidad peruana, por lo que es conveniente realizar ensayos adicionales con drogas afines que podrían destruir las formas intraepiteliales del parásito $\mathrm{y}$, así, ser usadas como medicamentos preventivos en la sarcocistiosis intestinal.

Los resultados obtenidos utilizando sulfadoxina y pirimetamina fueron negativos, a diferencia de lo hallado por Saravia (2003), quien en una infección experimental de perros con microquistes de $S$. lamacanis logró una eficacia del $100 \%$ al día 9 de tratamiento, aunque utilizando dosis más altas que las empleadas en el presente estudio. Yujra (2004) también reporta resultados satisfactorios al $6^{\circ}$ día del tratamiento, aunque no menciona las dosis empleadas.

\section{Conclusiones}

- El toltrazuril controló la sarcocistiosis a partir del $6^{\circ}$ día de iniciado el tratamiento.

- La combinación sulfadoxina y pirimetamina no pudo controlar la sarcosistiosis canina.

\section{Agradecimiento}

Un agradecimiento especial a INCAGRO, por el apoyo económico en la realización del presente proyecto, enmarcado dentro del subproyecto «Estrategia de manejo genético, sanitario y nutricional en la expresión de la capacidad genética de llamas para producción de carnes». Así como al Dr. Mario García por el apoyo incondicional en la corrección del presente artículo.

\section{Literatura Citada}

1. Bustinza, J. 2000. Enfermedades de las alpacas. $2^{\text {da }}$ ed. Akuarella Ed. p 110. Arequipa, Perú.
2. Corrales, A. 2000. Tasa de sarcosporidiosis de alpacas en los mercados de la avenida Andrés Avelino Cáceres, distrito de José Luis Bustamante y Rivero, Arequipa. Tesis de Médico Veterinario Zootecnista. Univ. Católica de Santa María. Arequipa. 55 p.

3. Daniel, D. 1996. Bioestadística base para el análisis de las ciencias de la salud. $5^{\text {a }}$ ed. Uteha. p 138-170. Mexico DF.

4. Daugschies, A; H. Mundt; V. Letkova. 2000. Toltrazuril treatment of cystoisosporosis in dogs under experimental and field conditions. Parasitol. Res. 86: 797-799.

5. Franco, E.; W. García; D. Pezo. 1998. Manual de crianza de llamas. Pub. Tec. IVITA No 33.42 p.

6. [INIA] Instituto Nacional de Investigación y Extensión Agraria. 2007. Camélidos [Internet] [acceso 01 junio 2007]. Disponible en: www.inia.gob.pe/ Camelidos/resumen.htm

7. Kassai, T. 1988. Helmintología veterinaria. p 68. Ed. Acribia. Zaragoza.

8. Leguía, G. 1991. The epidemiology and economic impact of llama parasites. Parasitol. Today 7: 54-56.

9. Leguía, G.; N. Clavo. 1989. Sarcocistiosis o triquina. Bol. Tec. IVITA $\mathrm{N}^{\mathrm{o}} 7.19 \mathrm{p}$.

10. Leguía, G.; Casas, E. 1999. Enfermedades parasitarias y atlas parasitológico de camélidos sudamericanos. p 23-34. Ed. De Mar. Lima.

11. Lindsay, D.; J. Dubey; T. Kennedy. 2000. Determination of the activity of ponazuril against Sarcocystis neurona in cell cultures. Vet. Parasitol. 92: 165169.

12. Lloyd, S.; J. Smith. 2001. Activity of toltrazuril and diclazuril against Isospora species in kittens and puppies. Vet. Record. 21: 509-511.

13. Madison, J.E.; S.W. Page; D. Church. 2004. Farmacología clínica en pequeños animales. p 174-175. Ed. Intermédica. Buenos Aires. 
14. Marthis, G.F.; R. Froyman; T. Kennedy. 2004. Coccidiosis control by administering in the drinking water for 2-days period. Vet. Parasitol. 121: 1-9.

15. Mostajo, W. 1983. Sarcocistiosis en alpacas beneficiadas en el camal municipal de Santa Rosa. Tesis de Médico Veterinario Zootecnista. Facultad de Medicina Veterinaria y Zootecnia. Univ. Nacional del Altiplano. Puno. $68 \mathrm{p}$.

16. Mundt, H.; C.A. Daugschies; F. Webe; M. Rinke. 2003. Efficacy of toltrazuril against infections with Eimeria bovis in calves. Parasitol. Res. 90: S166S167.

17. Saravia, Z. 2003. Anticoccidiales en el tratamiento de la sarcocistiosis en perros infectados con microquistes de alpaca. Tesis de Médico Veterinario y Zootecnista. Facultad de Medicina Veterinaria y Zootecnia, Univ. Nacional del Altiplano. $65 \mathrm{p}$.

18. Vilca, J.; F. Vilca; A. Chávez; M. Urviola; V. Leyva. 2007. Efecto del Toltrazuril al $2.5 \%$ durante el periodo prepatente de la sarcocistiosis intestinal canina. Rev. Inv. Vet., Perú 18: 64-68.

19. Yujra, F. 2004. Anticoccidiales en el tratamiento de sarcocistiosis en perros infectados con macroquistes de alpaca. Tesis de Médico Veterinario y Zootecnista. Facultad de Medicina Veterinaria y Zootecnia, Univ. Nacional del Altiplano. Puno. 74 p. 